\title{
Patient-Centered Development of a Digital Care Pathway for Arrhythmia Patients
}

\author{
Pia LILJAMO ${ }^{\mathrm{a}, 1}$ Hanna SÄILYNOJA $^{\mathrm{a}}$, Kirsi TUOMIKOSKI ${ }^{\mathrm{a}}$, \\ Anja HENNER ${ }^{\mathrm{b}}$ and Kirsi KOIVUNEN ${ }^{\mathrm{b}}$ \\ ${ }^{a}$ Oulu University Hospital, Northern Ostrobothnia District, Oulu, Finland \\ ${ }^{\mathrm{b}}$ Oulu University of Applied Sciences, Oulu, Finland
}

\begin{abstract}
Citizens are ready and willing to use various kinds of e-health services and Web-based portals. The purpose of this study was to describe the experiences of patients who underwent an arrhythmia procedure of the guidance they received as well as their needs and expectations for a future digital care path. The goal for the future is to utilize the results in other patient-centered digital service development activities. The research material was collected in a two-part thematic interview with patients who underwent an electrophysiology examination and supraventricular tachycardia catheter ablation procedure $(\mathrm{n}=7)$ or ablation treatment for atrial fibrillation $(n=4)$. The preliminary digital care path was modified based on the results. The arrhythmia patient's digital care path was tested in a workshop using a test group consisting of patients $(\mathrm{n}=3)$ and nursing staff $(\mathrm{n}=6)$. As a result, a digital care pathway for arrhythmia patients was completed.
\end{abstract}

Keywords. Arrhythmia, digitalization, eHealth, patient guidance

\section{Introduction}

In many countries, digital technologies are expected to bridge the rapidly growing gap between healthcare service demand and capacity. There are increasing demands for healthcare systems to shift to supporting consumers and patients in managing their own health and wellbeing. Digital services are becoming a recognized and integral part of all healthcare services $[1,2]$. Citizens are ready and willing to use different e-health services and Web-based portals. [3-5]. At this point, patients and citizens have decades of experience in using the Internet to search for health-related information. Even though patients trust healthcare professionals, they want look up symptoms on the Internet because information is easily accessible [6]. Patient portals are promising instruments for improving patient-centered care, as they provide patients with information and tools to better manage their health. The implementation of portals in both inpatient and outpatient settings gives health care providers more opportunities to support patients during hospitalization and after discharge [5]. Healthcare professionals are also optimistic about patient portals, provided that they are adequately informed in advance and that their organization is able to implement them well [7].

\footnotetext{
${ }^{1}$ Corresponding Author, Pia Liljamo, Oulu University Hospital, P.O. Box 1090029 OYS, Oulu, Finland, E-mail: pia.liljamo@ppshp.fi.
} 
There are many different types of e-health services in use globally, many designed to support self-medication of chronic diseases [8]. Chronic disease self-care e-services have brought benefits to patients [9]. In Denmark, the Active Heart portal was developed for self-treatment of cardiovascular disease, and patient experiences have been positive [10]. Mobile applications developed for atrial fibrillation patients facilitate communication between patients and professionals, increase patient participation in treatment decisions, and encourage self-care [11]. Mobile applications significantly improved patient informedness, drug adherence, satisfaction with anticoagulation therapy, and quality of life [12]. The use of mobile health applications has a positive effect on interaction between patients and healthcare providers, which correlates with better health outcomes and supports patient self-care [13].

A digital care pathway (DCP) is a secure digital service channel for patients in a care relationship with a specialized health care hospital in Finland. It is part of the Health Village portal built as a cooperative effort of five Finnish university hospitals, led by Helsinki University Hospital, within the Virtual Hospital project [3,14]. The DCP application enables patients to interact online with professionals and receive information about specific illnesses or symptoms and help for self-care. They can also access frequently asked questions, various exercises, questionnaires and a tool for monitoring personal health data. Accessing the DCP requires strong identification using e-banking identifiers or mobile ID. A doctor's referral or an existing care relationship is required [14]. The Virtual Hospital project established an e-health Development Model, which includes training and material to develop new CDPs. By the end of June 2020, eleven DCPs for different patient group had been implemented in Oulu University Hospital, with many more on the way. The goal of all DCPs is patient-oriented development, but with the arrhythmia path, patients were systematically involved from the outset.

The aim of this study was to describe the experiences of patients who underwent an arrhythmia procedure of the guidance they received, as well as their needs and expectations regarding the content of the future digital care path. Looking forward, the aim is to standardize patient-centered guidance material for patients entering an arrhythmia care procedure and utilize the results to make the e-health Development Model more patient-oriented.

\section{Methodology}

The topic of the second author's developmental research thesis was chosen so that it is adjacent to both practical nursing and development work, thus harnessing existing professional competence to the best effect in the development work [15]. Research material was collected through thematic interviews. The study utilized service design methods and a qualitative research approach. The research material was analyzed using content analysis. The research was conducted in the following main phases. The definition phase described the current method of treating arrhythmia patients, and outlined the content of the DCP and its proposed integration to the care protocol. The research phase incorporated the experiences of patients, who underwent an arrhythmia procedure of the guidance they received, as well as their needs and expectations regarding the content of the future DCP. The material for this phase was collected in a two-part thematic interview with patients who underwent an electrophysiology examination and supraventricular tachycardia (SVT) catheter ablation procedure $(n=7)$ and patients who underwent ablation treatment for atrial fibrillation $(n=4)$. The age of 
the patients interviewed ranged from 24 to 70 years and the sample included both males $(n=6)$ and females $(n=5)$. The thematic interview was done in two phases. The first interview was conducted after the procedure while the patients were still in hospital, and the second over the phone about a week after the procedure. Telephone interviews were appropriate because of long distances between home and hospital. The aim of the interview was to obtain information about the guidance received at discharge and how it helped patients to cope at home. The thematic interview gathered patient experiences of guidance at different stages of the treatment path, including guidance from the referring physician, written guidance in the appointment letter, and a pre-call two weeks before the operation, hospitalization, follow-up after the operation, discharge, and aftercare at home. The researcher emphasized that she did not work in that unit and the content of the interview had no effect on patients' care.

The preliminary DCP was modified based on the study results during the development phase. The arrhythmia patient DCP was further tested using a test group composed of patients who underwent an arrhythmia procedure $(n=3)$ and nursing staff from the Cardiology and Medical Day Wards $(n=6)$. After testing the DCP, feedback and development ideas, including a written review by one patient, were collected in a workshop. The workshop was recorded and the material studied using content analysis, after which the material of the DCP was modified into its final form.

\section{Results}

Patients' experiences of the traditional guidance they received varied. Patients felt that the guidance provided by the referring physician was inadequate and poorly applicable to their own situation, arrhythmias, and prognosis. Information about treatment options, the operation and associated risks was perceived as insufficient. The content of the appointment letter was generally considered clear and informative enough, but some felt that all necessary instructions should have been included in the appointment letter alone. Some patients were confused when the pre-call came before other information, and the guidance received over the phone was considered difficult to absorb. More guidance, partly the same as during the pre-call, was given at admission to the hospital. The guidance given during the procedure itself was good, but some would have liked more information in advance. Guidance on coping at home was generally considered adequate, but there were patients who felt uncertain what to do if symptoms occurred. Two of the interviewed patients said they preferred a traditional control model. The reason was that they did not own a computer or a smartphone or use the Internet. According to one patient, the traditional way is easy: instructions arrive by mail and the caregiver calls you, so you do not have to search for information. Although the call was well regarded, nine out of 11 patients would have been willing to try the DCP. The digital service sought to preserve what patients preferred in the traditional guidance. Many were pleased with the content of the appointment letter and hoped the path would contain the same information. The path should have preparatory instructions and an electronic pre-information form, as well as content related to aftercare and recovery, and track the patient's post-operation sensations. A more detailed description of the operation and the associated risks was requested, as was a FAQ section.

Based on the analysis of the interview material, the preliminary DCP for arrhythmic patients was modified. The patients interviewed felt that guidance was generally good, but a lack of information was felt at each stage of the care pathway. Especially at the 
referral phase, patients experienced deficiencies in guidance. Patients craved information about arrhythmia, its prognosis, treatment options, and the planned operation. Feelings of anxiety, as reported by the patients, were most prevalent at the time of arrhythmia diagnosis, but subsided as they learned more about their condition. As requested by the patients, the content of the DCP sought a clear and simple writing style, avoiding medical terminology. The structure of the path was divided into preparatory instructions, description of the procedure, and aftercare. The associated risks were placed under their own heading so that the patient knew them before entering the hospital. Patients wanted photographs and videos of the operation as well as anatomical drawings of the structure of the heart. Based on the analysis of the workshop work, the development worker compiled a summary of the test patients' requested improvements to content, visuality and usability using photos, text sequencing, and highlight boxes. The cardiology unit staff completed the final DCP and it will go into pilot operation in August 2020.

\section{Discussion}

In this process, the results of involving patients closely in the development of the DCP for arrhythmia patients are encouraging and productive. Patients were willing to participate in the process and, according to the answers, interested in using a digital service, equally in previous research [3-5]. Many patients felt that the DCP was a good addition to current services. Good knowledge of one's own arrhythmia and its treatment promotes patients' ability to influence their own care [13]. Health Village emphasizes the active role and equality of citizens in promoting their own wellbeing by implementing online and digital self-care services as part of the care process [3]. In the case studied here, patients wanted to have increased, complete, and timely information about their illness. Some, lacking technological competencies, still preferred the traditional information letter by mail. Experience with web portals, e.g. using a cardiac telerehabilitation web portal, can be beneficial for patient education and may increase patients' eHealth literacy skills [10]. Even though patients trust their physicians and their expertise, many prefer the Internet because it provides easy access to information [6]. Interviews and workshops revealed that in addition to text, the material of a DCP should include images and videos. The absorption and recall of patient guidance can be improved by using a variety of guidance materials [16]. An important addition was a video where a patient who had undergone the same procedure shared their experience; there is evidence that peer messaging in guidance reduces patient anxiety [17].

Digital services are expected to improve patient access to care and facilitate the workflow of healthcare professionals. Expectations for the cost-effectiveness and impact of digital transactions are high [3]. New e-health solutions must provide evidence-based benefits and be safe to use, and their impact on patients and organizations needs to be clarified and evaluated [1,2]. Recent studies [e.g. 18] show that an organization's view of the health services and care can differ in many ways from patients' experiences. Patients are experts in their own well-being and therefore an important resource in the development of care. Because the study was qualitative and participants were selected non-randomly, based on their willingness to cooperate, the results cannot be generalized. However, the goal of this study was to provide a rich, contextualized understanding of arrhythmia patients' experience through the intensive study of particular cases. Through this study, we gained evidence and experience on how to involve patients more systematically in the development of DCP. 


\section{Conclusion}

The content of the digital care pathway for arrhythmia patients was produced in collaboration with patients and caregivers. Patient experiences and suggestions for the guidance material were central to the result. These patient-centered methods can be utilized in the development of digital pathways for other patient groups. By involving patients in the development, the quality of service and commitment can be promoted.

\section{References}

[1] Ministry of Social Affairs and Health, Finland. Information to support well-being and service renewal. eHealth and eSocial Strategy 2020. Available in Finnish: http://urn.fi/URN:ISBN:978-952-00-3548-8.

[2] Schofield P, Shaw T, Pascoe M. Toward Comprehensive Patient-Centric Care by Integrating Digital Health Technology With Direct Clinical Contact in Australia. J Med Internet Res 2019;21[6]:e12382.

[3] Saranto K, Kivekäs E, Kuosmanen P, Kinnunen U-M. Electronic Health Services in the Patients' Daily Activities - Willingness to Use Health Village Services. Stud Health Technol Inform. 2018; 247:586-90. Doi: 10.3233/978-1-61499-852-5-586

[4] Hyppönen H, Aalto AM. Citizens experiences of e-health and e-welfare services. In: Vehko T, Ruotsalainen S, Hyppönen H (Eds). E-health and e-welfare of Finland. Checkpoint 2018. National Institute for Health and Welfare [THL], Helsinki, Finland. p. 148-167. Available: http://urn.fi/URN:ISBN:978-952-343-326-7

[5] McAlearney AS, Sieck CJ, Gaughan A, et al. Patients' Perceptions of Portal Use Across Care Settings: Qualitative Study. J Med Internet Res 2019;21[6]:e13126.

[6] Champlin S, Mackert M, Glowacki EM, Donovan EE. Toward a Better Understanding of Patient Health Literacy: A Focus on the Skills Patients Need to Find Health Information. Qual Health Res. 2017;27(8):1160-1176. doi:10.1177/1049732316646355

[7] Kujala S, Hörhammer I, Kaipio J, Heponiemi T. Health professionals' expectations of a national patient portal for self-management. Int J Med Inform. 2018;117:82-87. doi:10.1016/j.ijmedinf.2018.06.005

[8] Rush KL, Hatt L, Janke R, Burton L, et al. The efficacy of telehealth delivered educational approaches for patients with chronic diseases: A systematic review. Patient Educ Couns. 2018;101(8):1310-1321.

[9] Neubeck L, Coorey G, Peiris D, et al. Development of an integrated e-health tool for people with, or at high risk of, cardiovascular disease: The Consumer Navigation of Electronic Cardiovascular Tools [CONNECT] web application. Int J Med Inform. 2016; 96:24-37. doi:10.1016/j.ijmedinf.2016.01.009

[10] Melholt C, Joensson K, Spindler H, et al. Cardiac patients' experiences with a telerehabilitation web portal: Implications for eHealth literacy. Patient Educ Couns. 2018;101(5):854-861.

[11] Kotecha D, Chua WWL, Fabritz L, et al., European Society of Cardiology (ESC) Atrial Fibrillation Guidelines Taskforce, the CATCH ME consortium and the European Heart Rhythm Association (EHRA). European Society of Cardiology smartphone and tablet applications for patients with atrial fibrillation and their health care providers. EP Europace, 2018; Feb. 20(2):225-33.

[12] Guo Y, Lane DA, Wang L, Chen Y, Lip GYH; mAF-App II Trial investigators. Mobile Health (mHealth) technology for improved screening, patient involvement and optimising integrated care in atrial fibrillation: The mAFA (mAF-App) II randomised trial. Int J Clin Pract. 2019;73(7):e13352.

[13] Qudah B, Luetsch K. The influence of mobile health applications on patient - healthcare provider relationships: A systematic, narrative review. Patient Educ Couns. 2019;102(6):1080-1089.

[14] Digital Health Village. https://www.digitalhealthvillage.com/en/home [Accessed on $15^{\text {th }}$ of July, 2020].

[15] Säilynoja H. Development of a digital treatment path for an arrhythmia patient at the Department of Cardiology, Oulu University Hospital. Oulu University of Applied Sciences Master's Degree Program in Development and Management of Health and Social Care. 2020. Available: http://urn.fi/URN:NBN:fi:amk-202005098116.

[16] Villanueva C, Talwar A, Doyle M. Improving informed consent in cardiac surgery by enhancing preoperative education. Patient Educ Couns. 2018; 101(x):2047-53.

[17] Salmasi S, Kwan L, MacGillivray J, et al. Assessment of atrial fibrillation patients' educations need from patient and clinician perspectives: A qualitative descriptive study. Thromb. Res. 2019;173:109-16.

[18] Bombard Y, Baker G.R, Orlando E. et al. Engaging patients to improve quality of care: a systematic review. Implementation Sci 2018; 13, 98. https://doi.org/10.1186/s13012-018-0784-z 